D.C. Rung

Nagoya Math. J.

Vol. 33 (1968), 75-83

\title{
SOME CONDITIONS UNDER WHICH SEQUENCES OF FUNCTIONS ARE UNIFORMLY BOUNDED
}

\author{
D.C. RUNG
}

\section{§1. Introduction}

After one introduces the theory of normal families in a course in complex analysis, the usual pattern is to give an example of a non-normal family. One of the simplest, of course, is the sequence $f_{n}(z)=n z, n=1,2, \cdots$. The very devastating effect of multiplying by zero insures the required abnormality! If one asks for a slightly more sophisticated example, we offer $f_{n}(z)=\frac{e^{n z}}{n}$, $n=1,2 \cdots$; here the $f_{n}(z)$ are zero free. However, the difference in behavior between the sequence $\left\{\left|f_{n}(0)\right|=\frac{1}{n}\right\}$ and $\left|f_{n}(z)\right|=\frac{e^{n x}}{n} z=x+i y \neq 0$ is obvious.

In this paper we offer an explanation for this state of affairs. To be precise given a sequence $\left\{f_{n}\right\}$ of bounded holomorphic functions defined in the unit disk $D$ in the complex plane we establish criteria based upon a comparison of $\left\{\left|f_{n}(0)\right|\right\}$ and $\left\{M\left(f_{n}\right)\right\}, M\left(f_{n}\right)=\max _{|z|<1}\left|f_{n}(z)\right|$ insuring that the sequence $\left\{f_{n}\right\}$ will be uniformly bounded on certain compact subsets of $D$. We then extend the result in several directions to entire and harmonic functions.

Let $D(r), 0<r<\infty$, denote the open disk in the complex plane with centre at the origin and radius $r$, while $\bar{D}(r)$ indicates the closure of $D(r)$; and $D(\infty)$ indicates the finite complex plane. If $f$ is a complex valued function defined in $D(R)$, for $0 \leq r \leq R$ let

$$
M(r, f)=\sup _{|z|<r}|f(z)|
$$

and

$$
m(r, f)=\inf _{|z|<r}|f(z)|
$$

If $E$ is a set contained in $D(R)$ set

Received January 11, 1968. 
If we need not display the dependence on $f$ we write $M(r)$, and $m(r) M_{E}(r)$ respectively.

\section{§2. Basic Theorem}

The results of this paper hinge on the solution of the Carleman-Milloux problem which we give as formulated by Tsuji [1 p. 307]. For this formulation we suppose that $E$ is a set contained in $D(R), 0<R<\infty$, with the properties that

i) each circle $|z|=r, 0<r<R$, meets $E$;

ii) $E \cap \bar{D}(r)$ is a closed set for each $0<r<R$.

We call such a set an intersecting set.

Theorem A. Let $E$ be an intersecting set in $D(R)$ and $f$ be holomorphic in $D(R)-E$, with $|f(z)| \leq M, z \in D(R)-E$, and

$$
\varlimsup_{\substack{z \rightarrow E \\ z \in D(R)-E}}|f(z)| \leq m<M
$$

Then for $z \in D(R)-E$

$$
\log |f(z)| \leq \frac{2}{\pi}\left(\arcsin \frac{R-|z|}{R+|z|}\right) \log m+\left(1-\frac{2}{\pi} \arcsin \frac{R-|z|}{R+|z|}\right) \log M
$$

Actually this is not precisely the formulation of Tsuji who requires that $E$ be closed in $\bar{D}(R)$. However it is evident that if one applies Tsuji's formulation to the disk $D(r), 0 \leq r<R$, and let $r \rightarrow R$ one obtains Theorem A.

We now look for functions $f$ with intersecting sets on which $f$ is bounded by some useful number. There is one rather simple condition which insures the existence of an interesting intersecting set and it is that $f$ takes on some finite value $\alpha$ only a finite number of times. Before giving a formal statement of this fact we introduce some standard notation.

If $f$ takes on the value $\alpha$ only a finite number of times in $D(R)$, $0<R<\infty$, let $\left\{z_{i}\right\}_{i=1}^{n}$ be the non-zero points, counted with proper multiplicity, at which $f\left(z_{i}\right)=\alpha$; and let $z=0$ be a root of $f(z)-\alpha$ of multiplicity $p \geq 0$ where $p=0$ means $f(0) \neq \alpha$. 
Set

$$
B_{\alpha}(z)=\frac{z^{p}}{R^{p}} \prod_{i=1}^{n} \frac{R z_{i}}{\left|z_{i}\right|}\left(\frac{z_{i}-z}{R^{2}-\bar{z}_{i} z}\right) .
$$

Lemma 1. Suppose $f$ is holomorphic in $D(R), 0<R<\infty$, and there takes on the value $\alpha$ only a finite number of times. Then the set

$$
E_{\alpha}=\left\{z \in D(R)|| f(z)|\leq| \frac{f(0)-\alpha}{B_{\alpha}(0)}|+| \alpha \mid \equiv M_{\alpha}\right\}
$$

is an intersecting set.

Proof. We first consider the case in which $f$ omits the value 0 . That $E_{0}=\{z \in D(R)|| f(z)|\leq| f(0) \mid\}$ is an intersecting set can be seen in several ways. We can use Cauchy's integral formula as follows: Suppose $E_{0}$ does not meet some circle $|z|=r_{0}, 0 \leq r_{0}<R$. Since $\frac{1}{f}$ is also holomorphic in $D(R)$

$$
\begin{aligned}
\left|\frac{1}{f(0)}\right| & \leq \frac{1}{2 \pi} \int_{0}^{2 \pi} \frac{d \theta}{\left|f\left(r_{0} e^{i \theta}\right)\right|} \\
& <\frac{1}{2 \pi} \int_{|z|=r_{0}} \frac{d \theta}{|f(0)|}=\frac{1}{|f(0)|},
\end{aligned}
$$

which is absurd. Since $E_{0} \cap\{|z| \leq r\}, 0 \leq r<R$, is a closed set $E_{0}$ is intersecting.

For future exploitation we observe that if $R=\infty$ and $f$ is an entire function which omits 0 then $E_{0}$ is also an intersecting set relative to $D(\infty)$.

For the general situation in which $f$ takes on $\alpha$ a finite number of times in $D(R), 0<R<\infty$, we let $g(z)=\frac{f(z)-\alpha}{B_{\alpha}(z)}$, which omits the value 0 . Therefore

$$
E_{0}{ }^{*}=\{z \in D(R)|| g(z)|\leq| g(0) \mid\}
$$

is intersecting. Now for $z \in E_{0}^{*}$,

$$
\begin{aligned}
|g(0)| & =\left|\frac{f(0)-\alpha}{B_{\alpha}(0)}\right| \geq|g(z)| \\
& =\left|\frac{f(z)-\alpha}{B_{\alpha}(z)}\right| \\
& \geq|f(z)|-|\alpha| .
\end{aligned}
$$


Hence the set $E_{\alpha}$ contains $E_{0}{ }^{*}$ which implies that $E_{\alpha}$ is also intersecting and the lemma is proved.

\section{§3. Applications to functions omitting the value 0 .}

In this section we assume that the sequence of function $\left\{f_{n}\right\}$ all omit the value 0 .

For $0<\delta<\infty$, let $B(\delta)=\sin \frac{\pi}{2(1+\delta)}$. An easy calculation gives that the subset of $D(R), 0<R<\infty$, given by $\frac{2}{\pi} \arcsin \frac{R-|z|}{R+|z|}>\frac{1}{1+\delta}$ is the disk $|z| \leq R\left(\frac{1-B(\delta)}{1+B(\delta)}\right)$. With this observation and the fact, already noted, that for a holomorphic function $f$ omitting 0 in $D(R)$ the set

$$
E_{0}=\{z \in D(R)|| f(z)|\leq| f(0) \mid\}
$$

is intersecting in $D(R)$ we use Theorem $A$ to give the key theorem.

Theorem I. Let $f$ be holomorphic and bounded in $D(R), 0<R<\infty$, and omit there the value 0 . Then for $|z|<R$ we have

$$
|f(z)| \leq|f(0)|^{\frac{2}{\pi} \arcsin \frac{R-|z|}{R+|z|}}(M(R, f))^{\left(1-\frac{2}{\pi} \arcsin \frac{R-|z|}{R+|z|}\right)}
$$

and thus for $|z|<R\left(\frac{1-B(\delta)}{1+B(\delta)}\right)$

$$
|f(z)| \leq|f(0)|^{\frac{1}{1+\delta}}(M(R, f))^{\frac{\delta}{1+\delta}} .
$$

Proof. Since $|f(z)|$ is bounded by $|f(0)|$ on the intersecting set $E_{0}$ a direct application of Theorem A gives (3.0). The convexity of the right side of (3.0) together with the remarks preceding this theorem validate (3. 1).

By way of illustration, if $f$ is holomorphic, bounded by 1 in $D(1)$, and omits 0 there, then

$$
|f(z)| \leq \sqrt[n+1]{|\bar{f}(0)|}, \quad|z| \leq\left(\frac{1-B(n)}{1+B(n)}\right) .
$$

There are some obvious results to be gathered from Theorem I about sequences of holomorphic functions omitting zero.

Corollary 1. Let $\left\{f_{n}\right\}$ be a sequence of bounded holomorphic functions in $D(R)$ with each $f_{n}$ omitting the value 0 . If for $\delta>0$ 


$$
\left|f_{n}(0)\right|\left(M\left(R, f_{n}\right)\right)^{\grave{o}} \leq A, \quad n=1,2, \cdots,
$$

then for

$$
\begin{aligned}
& |z| \leq\left(\frac{1-B(\delta)}{1+B(\delta)}\right) R, \\
& \left|f_{n}(z)\right| \leq A^{1+\delta}, n=1,2, \cdots
\end{aligned}
$$

Proof. This is an immediate application of Theorem I.

One can avoid the hypothesis that each $f_{n}$ be bounded.

Corollary 2. Let $\left\{f_{n}\right\}$ be a sequence of holomorphic functions in $D(R)$, each omitting the value 0 . If, for some sequence $\left\{r_{n}\right\}, 0<r_{n}<r_{n+1}<R, r_{n} \rightarrow R$, $n \rightarrow \infty$, and some value $0<\delta<\infty$,

$$
\left|f_{n}(0)\right|\left(M\left(r_{n}, f_{n}\right)\right)^{\delta} \leq A, \quad n=1,2, \cdots,
$$

then for

$$
\begin{gathered}
|z| \leq\left(\frac{1-B(\delta)}{1+B(\delta)}\right) r_{n}, \quad n=1,2, \cdots, \\
\left|f_{n}(z)\right| \leq A^{1+o} .
\end{gathered}
$$

Proof. Setting $g_{n}(\xi)=f_{n}\left(\begin{array}{l}r_{n}-\xi \\ R\end{array}\right)$ and invoking Corollary 1 gives this result.

We now make several comments on Corollary 1. That each $f_{n}$ must omit zero is needed. If we let $\delta=1, R=1$, and set $f_{n}(z)=n\left(z+\frac{1}{n^{2}}\right)$, $n=1,2, \cdots$, then $f_{n}(0)=\frac{1}{n}$ while $M\left(1, f_{n}\right)=n+\frac{1}{n}$. Thus $\left|f_{n}(0)\right| M\left(1, f_{n}\right)$ $\leq 2$, but the sequence is not uniformly bounded in any $D(r)$, $0<r<1$. On the other hand the example given in the introduction $f_{n}(z)=\frac{e^{n z}}{n}$ is without zeroes for each $n=1,2, \cdots ;$ has $\left|f_{n}(0)\right| M\left(1, f_{n}\right) \rightarrow \infty$, and is not uniformly bounded on any subdisk $|z|<r, 0<r<1$.

If $\left\{f_{n}\right\}$ is a sequence satisfying the hypothesis of Corollary 1 , with $\delta=1, R=1$, we are guaranteed by this result that on the closed disk $D\left(\frac{1-B(1)}{1+B(1)}\right)$ the sequence $\left\{f_{n}\right\}$ is uniformly bounded. We ask for the largest possible closed disk $\bar{D}\left(R^{*}\right)$ on which any sequence satisfying the hypothesis of Corollary 1 (with $\delta=1$ ) is uniformly bounded. We do not know the exact value of $R^{*}$. The sequence

$$
f_{n}(z)=2^{\frac{-n}{2}}(z+1)^{n}, \quad n=1,2, \cdots,
$$


satisfies the hypotheses of Corollary 1. Further $\left|f_{n}(z)\right| \leq 1$, for $|z| \leq(\sqrt{2}-1)$; but for any $\varepsilon>0$

$$
f_{n}(\sqrt{2}-1+\varepsilon) \rightarrow \infty
$$

so that we can only estimate that

$$
\frac{1-B(1)}{1+B(1)}=(\sqrt{2}-1)^{2} \leq R^{*} \leq(\sqrt{2}-1)
$$

Suppose again that $\left\{f_{n}\right\}$ is a sequence of holomorphic functions in $D(R)$, each of which omits the value 0 . Suppose further that for each $0<\delta<\infty$ there is a sequence $\left\{r_{n}\right\}, r_{n} \rightarrow R$, (with $\left\{r_{n}\right\}$ depending on $\delta$ ) such that $\varlimsup_{n \rightarrow \infty}\left|f_{n}(0)\right|\left(M\left(r_{n}, f_{n}\right)\right)^{\delta}<\infty$, then $\left\{f_{n}\right\}$ form a normal family. That this is so follows from Corollary 5. Given any disk $D(r), 0<r<R$, choose a value $\delta$ and an $N_{0}$ so that $r<\left(\frac{1-B(\delta)}{1+B(\delta)}\right) \frac{R^{2}}{r_{n}}, n>N_{0}$. Then $\left\{f_{n}\right\}$ is uniformly bounded in $D(r)$ and so is a normal family.

In passing we mention that Corollary 1 can be used to give a proof of Hurwitz's theorem. Suppose $\left\{f_{n}\right\}$ is a sequence of holomorphic functions defined, for simplicity, in $D(1)$ such that $\left\{f_{n}\right\}$ converges uniformly (in the spherical metric) on compact subsets of $D(1)$. Suppose $f_{n}(0) \rightarrow \alpha, n \rightarrow \infty$ but on some $D(r), 0<r<1$, no $f_{n}$ takes on the value $\alpha$. If $\alpha$ is finite, and since each $f_{n}$ is continuous, we can conclude that for some $0<r_{1}<r$, $\left\{f_{n}\right\}$ is uniformly bounded, say by $M$, on $D\left(r_{1}\right)$. According to Corollary 4, applied to $\left\{f_{n}-\alpha\right\}$ on $D\left(r_{1}\right)$

$$
\left|f_{n}(z)-\alpha\right| \leq \sqrt{\left|f_{n}(0)-\alpha\right| M,},|z| \leq(\sqrt{2}-1)^{2} r_{1} .
$$

Thus $f_{n}(z) \rightarrow \alpha$ for all $|z| \leq(\sqrt{2}-1)^{2} r_{1}$ and therefore the limit function is identically $\alpha$ in $D(1)$. In the situation for which $\alpha$ is infinite we consider $\left\{\frac{1}{f_{n}}\right\}$.

\section{§4. Bounded functions}

With little effort we can extend Theorem I to cover arbitrary bounded functions by invoking the usual factorization by a Blaschke product. For a given holomorphic function $f$ bounded by 1 in $D(1)$ let $\left\{z_{n}\right\}$ be the set of all non-zero roots of $f$ with each root repeated as often as its multiplicity. Also suppose $f$ has a zero of order $p$ at the origin (where $p=0$ means $f(0) \neq 0)$. Then the Blaschke product 


$$
B(z, f)=z^{p} \prod_{n=1}^{\infty} \frac{z_{n}}{\left|z_{n}\right|} \frac{z_{n}-z}{1-z}
$$

is known to converge in $D(1)$ to a holomorphic function and $f$ can be factored into $f=B g$, where $g$ is a holomorphic function in $D$ without zeros. Using this fact we give

Theorem II. Let $f(z)$ be a holomorphic function in $D(1)$ with

$$
|f(z)|<1, z \in D(1) .
$$

Then

$$
|f(z)| \leq|B(z ; f)||g(0)|^{\frac{2}{\pi} \arcsin \frac{1-|z|}{1+|z|}} .
$$

Proof. According to Theorem I applied to the function $g$ (and noting that $|g(z)|<1, z \in D(1))$

$$
|g(z)|<|g(0)|^{\frac{2}{\pi} \arcsin \frac{1-|z|}{1+|z|}} .
$$

Since $g=\frac{f}{B}$ the proof is complete.

\section{\$5. Entire functions omitting 0 .}

Our next application is to entire functions omitting 0 .

Theorem III. Suppose $f$ is an entire function omitting the value 0 such that for some sequence $\left\{r_{n}\right\}, \quad 0<r_{n}<r_{n+1}<\infty, \quad r_{n} \rightarrow \infty, n \rightarrow \infty$; some choice of $0<\delta<\infty$; and some arbitrary but fixed $T>\frac{2}{1-B(\delta)}$

$$
\lim _{n \rightarrow \infty} m\left(r_{n}, f\right)\left[M\left(T r_{n}, f\right)\right]^{\delta}<\infty .
$$

Then $f$ is a constant function.

Proof. For $n=1,2, \cdots$ let $\left|f\left(z_{n}\right)\right|=m\left(r_{n}, f\right)$; also set

$$
\xi=\xi_{n}(z)=\frac{z-z_{n}}{(T-1) r_{n}}
$$

and

$$
g_{n}(\xi)=f\left(\xi_{u}^{-1}(\xi)\right) \text {. }
$$

A trivial calculation shows that the disk about $z_{n}$ of radius $r_{n}(T-1)$ is 
contained in $D\left(T r_{n}\right)$. Consequently $M\left(1, g_{n}\right) \leq M\left(T r_{n}, f\right), n=1,2, \cdots$. Since $\left|g_{n}(0)\right|=\left|f\left(z_{n}\right)\right|=m\left(r_{n}, f\right)$ we conclude that

$$
\lim _{n \rightarrow \infty}\left|g_{n}(0)\right|\left(M\left(1, g_{n}\right)\right)^{\delta}<\infty .
$$

If we choose $A>0$ so that $\left|g_{n}(0)\right|\left(M\left(1, g_{n}\right)\right)^{\delta}<A$, all $n$, and notice that each $g_{n}(z)$ omits 0 , Theorem I allows us to conclude that for

$$
\begin{gathered}
|\xi| \leq \frac{1-B(\delta)}{1+B(\delta)}, n=1,2, \cdots, \\
\left|g_{n}(\xi)\right| \leq\left[\left|g_{n}(0)\right|\left(M\left(1, g_{n}\right)\right)^{\delta}\right]^{\frac{1}{1+\delta}} \leq A^{\frac{1}{1+\delta}} .
\end{gathered}
$$

Thus in this $\xi$ disk the sequence $\left\{g_{n}\right\}$ is uniformly bounded. But this says that $f$ is bounded by $A^{\frac{1}{1+\delta}}$ on each disk $\frac{\left|z-z_{n}\right|}{(T-1) r_{n}} \leq \frac{1-B(\delta)}{1+B(\delta)}, n=1,2, \cdots$. It can be seen that each of these disks contain the corresponding disk about the origin of radius $r_{n}\left[\frac{1-B(\delta)}{1+B(\delta)}(T-1)-1\right] \equiv r_{n} p$. However the quantity inside the brackets is seen to be fixed positive number on account of our choice of $T>\frac{2}{1-B(\delta)}$. Hence

for

$$
\begin{aligned}
& |f(z)| \leq A^{\frac{1}{1+\delta}} \\
& z \in \bigcup_{n=1}^{\infty} D\left(p r_{n}\right),
\end{aligned}
$$

which union covers the plane and so application of Liouville's theorem completes the proof.

Observe that as $\delta \rightarrow 0, \frac{2}{1-B(\delta)} \rightarrow+\infty$ and as $\delta \rightarrow \infty, \frac{2}{1-B(\delta)} \rightarrow 2$, so if one wants a smaller multiple of $r_{n}$ in computing the maximum modulus one must increase the exponent of the maximum modulus and vice-versa. If we take $\delta=1$ then $\frac{2}{1-B(1)}$ is about 6.82 so if $f$ is an entire function omitting 0 and

$$
\lim _{r \rightarrow \infty} m(r, f) M(7 r, f)<\infty
$$

then $f$ is a constant function.

\section{\$6. Harmonic functions}

By the usual device of calling forth the exponential function we can 
rework Theorem I to yield some results on harmonic functions. The theorem becomes

Theorem IV. Let $u(z)$ be harmonic and bounded above by $M$ in $D(R)$, $0<R<\infty$. Then for $|z| \leq R\left(\frac{1-B(\delta)}{1+B(\delta)}\right)$ we have

$$
u(z) \leq \frac{1}{1+\delta}[u(0)+\delta M]
$$

Proof. If we let $v$ be a complex conjugate of $u(z)$ and form $g=e^{u+i v}$ then $g$ is holomorphic and zero-free in $D(R)$. Hence Theorem I prevails and the result is immediate.

We have parallel results to Corollaries 1 and 2. We give only the analogous result to Corollary 1.

COROllary 3. Let $\left\{u_{n}\right\}$ be a sequence of bounded harmonic functions in $D(R), \quad 0<R<\infty$, such that for some $0<\delta<\infty \varlimsup_{n \rightarrow \infty}\left(u_{n}(0)+\delta M\left(R, u_{n}\right)\right)<\infty$. Then the sequence $\left\{u_{n}\right\}$ is uniformly bounded from above on $|z| \leq R\left(\frac{1-B(\delta)}{1+B(\delta)}\right)$.

Proof. This follows from Theorem IV in the same way Corollary 1 follows from Theorem I.

Remark. Generalizations of our theorems in the case $f$ (or $\left\{f_{n}\right\}$ ) take a value $\alpha$ only finitely often are possible by using the full force of Lemma 1. However the results seem a bit technical so we do not list them.

\section{Reference}

[ 1 ] M. Tsuji, Potential theory in modern function theory, Maruzen, Tokyo, 1959.

D.C. Rung

The Pennsylvania State University

University Park, Pennsylvania 